\title{
Takotsubo cardiomyopathy in two men receiving bevacizumab for metastatic cancer
}

\author{
Thérèse $\mathrm{H}$ Franco \\ Ahmed Khan \\ Vishal Joshi \\ Beje Thomas
}

Department of Internal Medicine, University of Connecticut, Farmington, CT, USA
Correspondence:Thérèse $\mathrm{H}$ Franco University of Connecticut, Department of Internal Medicine, 263 Farmington Avenue, Farmington, Connecticut, USA, 06030- 1234

Tel + I 8606794017

Fax +| 860679 |62।

Email tfranco@resident.uchc.edu

\begin{abstract}
Bevacizumab is a monoclonal antibody that inhibits vascular endothelial growth factor (VEGF). It is a novel chemotherapeutic agent currently approved as part of combination chemotherapy for metastatic colorectal cancer, non-small cell lung cancer, and breast cancer (Hurwitz et al 2004; Sandler et al 2006; Traina et al 2007). Arterial thrombosis, including cerebral infarction, transient ischemic attacks, myocardial infarction, and angina are common, occurring in $4.4 \%$ of patients whose regimen includes bevacizumab (versus $1.9 \%$ on regimen without bevacizumab) (Genetech, Inc. 2008). This series will review two cases of patients exposed to bevacizumab who subsequently developed ST elevations on electrocardiogram (ECG) and elevated cardiac biomarkers. Both patients underwent cardiac catheterization, which demonstrated apical ballooning and akinesis in a distribution discordant with the observed (noncritical) atherosclerotic lesions. Both patients had recovery of left ventricular function within 30 days. The clinical presentation, including ECGs and findings on catheterization as well as the rapid recovery of ventricular function, is consistent with the diagnosis of takotsubo cardiomyopathy. Takotsubo cardiomyopathy was first described in 1991, but the pathophysiology and exact mechanism of injury remain largely unknown. These two cases are notable for their occurrence in men and the association with treatment of metastatic cancer including bevacizumab.
\end{abstract}

Keywords: vascular endothelial growth factor, bevacizumab, metastatic cancer, chemotherapy, takotsubo, cardiomyopathy

\section{Introduction}

Takotsubo cardiomyopathy was originally described in 1991 by Dote and colleagues. The appearance of the apical ballooning on ventriculography (characterized by a round base and narrow neck) resembles a vessel used in Japan as an octopus trap. The vessel is called takot subo, thus, the syndrome named (Dote et al 1991). The takotsubo syndrome has garnered much attention with a series of case reports and reviews in the recent literature. Initially thought to be limited to Japan and geographically and/or racially selective, case reports in Caucasian populations throughout the United States and Europe have increased. It remains unclear whether this effect is due to a true increase in the incidence or if it is due to more widespread recognition of the syndrome.

Takotsubo syndrome is characterized by the abrupt onset of angina-like chest pain, ST elevations and/or T wave inversions, subsequent elevation of cardiac biomarkers, and a marked decrease in left ventricular systolic function. It occurs most commonly in women, aged 62 to 75 . Patients generally present with chest pain, but may also complain of dyspnea or rarely, syncope (Bybee et al 2004). Imaging of the ventricular function reveals a hyperdynamic basal segment and apical akinesis of the left ventricle, whereby takotsubo mimics acute myocardial infarction (Dote et al 1991; Bybee et al 2004).There remain, however, important differences between the two diagnoses. For example, in takotsubo cardiomyopathy, peak troponin values are often observed on initial presentation as opposed to the later peak as seen in myocardial infarction. 
Furthermore, if any coronary artery disease is discovered on angiogram, it is incongruent with the akinesis visualized. Finally, in takotsubo cardiomyopathy, resolution of the impaired left ventricular systolic function occurs in 7 to 30 days following presentation (Bybee et al 2004).

The pathogenesis of takotsubo cardiomyopathy is incompletely understood. Transient myocardial arterial thrombosis, with subsequent dislodgement of clot or plaque seems unlikely because of the absence of a plausible blockage of coronary arterial territory producing ischemia congruent with the area in which the akinesis is seen. Lymphocytic myocarditis is characterized by a reversible deficit of left ventricular output due to hypokinesis in areas extending beyond a single coronary artery. The hypokinesis seen in myocarditis, however, is global and does not have the characteristic apical ballooning seen with takotsubo. Coronary vasospasm without thrombosis is also a consideration; however, past studies have failed to determine clearly if vascular spasm is the direct mechanism of injury or if the vascular spasm is simply a secondary phenomenon in takotsubo cardiomyopathy (Bybee et al 2004). The most commonly accepted theory is that stress induced catecholamine release causes myocardial damage by microvascular spasm or by direct cardiotoxicity. Microvascular ischemia might account for the lack of a single coronary artery territory producing ischemia in the apical area of akinesis (Dote et al 1991; Bybee et al 2004). Adrenergic receptor density appears to be heterogeneous throughout the left ventricle, which might explain the regional variation in myocardial damage that is observed during the high catecholamine state. Parallels have been drawn to the myocardial stunning seen with subarachnoid hemorrhage and high levels of catecholamines found in pheochromocytoma. Genetic variations in catecholamine receptor location could place susceptible individuals at increased risk for this syndrome (Van De Walle et al 2006).

Although takotsubo syndrome is normally found in women, both patients in these cases were men (Dote et al 1991; Bybee et al 2004). Both patients received bevacizumab as chemotherapy for metastatic cancer. Bevacizumab is a vascular endothelial growth factor (VEGF) inhibitor. VEGF inhibition is a novel target for chemotherapy, and the potential utility of this class of drugs is quite large. While the role of VEGF in embryonic development and neoplastic processes is widely accepted, it is not clear what role this molecule might have in the adult vascular system. There is some suggestion that VEGF may perform arterioprotective functions (Zachary et al 2006). In an experiment designed to elucidate the molecular biology of cardiotoxicity secondary to chemotherapy, high levels of inflammatory cytokines and chemoattractant proteins were isolated in the plasma and myocardial tissue of mice that were exposed to bevacizumab. Researchers have proposed that the expression of these inflammatory cytokines and chemoattractant proteins in plasma during anti-cancer therapy could be indicative of cardiotoxicity (Drimal et al 2006). In an animal model of heart failure and pressure overload (with transaortic constriction), mice that were genetically engineered to block signaling of VEGF demonstrated a reduction in cardiac hypertrophy with increased left ventricle dilatation and a loss in contractile function. These findings suggest that inhibition of VEGF is associated with the transition from compensatory hypertrophy to overt failure (Izumiya et al 2006).

VEGF inhibition has also been associated with arterial thrombotic events. A recent meta-analysis, which included five randomized controlled trials, found that the risk of arterial thrombosis was higher in cancer patients receiving bevacizumab as part of their regimen, than in those patients whose regimen did not include bevacizumab (Scappaticci et al 2007). These findings are consistent with the statistics from prescribing information for bevacizumab. Arterial thrombotic events, such as myocardial infarction and angina, occurred in $4.4 \%$ of patients whose regimen included bevacizumab as opposed to $1.9 \%$ of patients on a regimen without bevacizumab (Genetech, Inc. 2008). Given the similarities between myocardial infarction and takotsubo cardiomyopathy, it is conceivable that some of the arterial thrombotic events recorded as myocardial infarction might actually have been unrecognized incidents of takotsubo cardiomyopathy.

There are currently 130 bevacizumab clinical trials for 25 different types of cancer. If there is direct cardiotoxicity or other deleterious vascular effects inherent in this class of drugs, the implications and number of patients affected are substantial.

\section{Cases}

In the first case, a 76-year-old man with colon cancer presented to the emergency room with fever and hypotension 2 days after initiating chemotherapy with bevacizumab. The ECG (Figure 1) demonstrated diffuse anterior and inferior ST-segment elevations in the setting of elevated troponin $(11.4 \mathrm{ng} / \mathrm{mL})$. Emergent cardiac catheterization revealed noncritical left main coronary artery disease and atherosclerosis in the right coronary artery (Figure 2a, 2b). Echocardiography showed decreased systolic function with apical akinesis and a hyperdynamic basal segment of the left ventricle, which was discordant with the findings at cardiac 


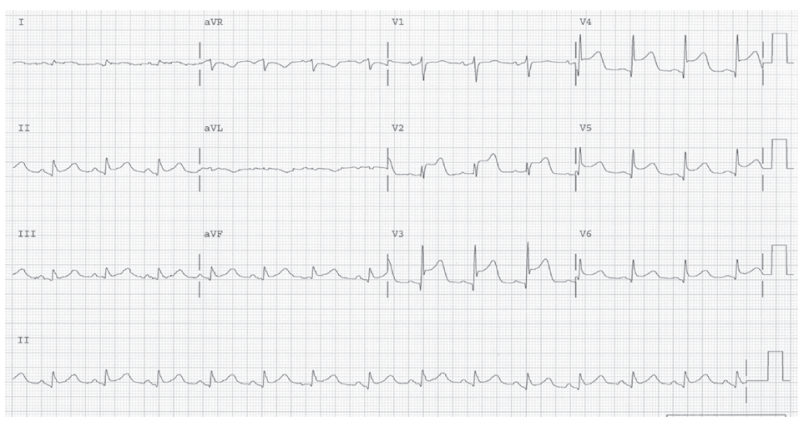

Figure I The ECG for case one demonstrated prominent ST elevations in the anterior and inferior leads.

catheterization. The patient's fever and hypotension subsided without intervention and no source of infection was identified. Approximately 3 weeks later, during a separate admission for lower gastrointestinal bleeding, minimally invasive hemodynamic and cardiac monitoring revealed normal cardiac output, consistent with recovery of left ventricular function.

In the second case, a 61-year-old man with metastatic non-small cell lung cancer presented to the emergency room with hemoptysis. The patient was tachycardic, with a heart rate of 100 , but normotensive with pulse oximetry of $98 \%$. Three weeks prior to admission, the patient had received the second dose of chemotherapy including bevacizumab. The ECG (Figure 3) demonstrated new Q-waves in the inferior and precordial leads, as well as diffuse ST elevation suggestive of acute ischemia. Creatine-kinase (CK) and CK-MB (CK-muscle and brain) were normal, but troponin was elevated $(2.5 \mathrm{ng} / \mathrm{mL})$. On cardiac catheterization, the patient had severely depressed left ventricular function with no evidence of coronary arterial stenosis (Figure 2c, 2d). The hemoptysis resolved and no further hemodynamic compromise was observed. An ECG on the third day of hospitalization showed resolution of the ST elevations, but the Q-waves persisted. An echocardiogram performed on day 15 of his hospitalization was notable for significant recovery of ventricular function.

\section{Discussion}

The differential diagnosis of acute myocardial infarction in the absence of critical coronary artery disease is broad. It includes cocaine intoxication, coronary vasospasm, prolonged hypotension, sepsis, micro-embolic occlusion, CNS trauma, vasculitis, coronary artery dissection, and takotsubo cardiomyopathy. After appropriate diagnostic evaluation, and in light of the fact that both patients met all four of the proposed Mayo Criteria for the clinical diagnosis

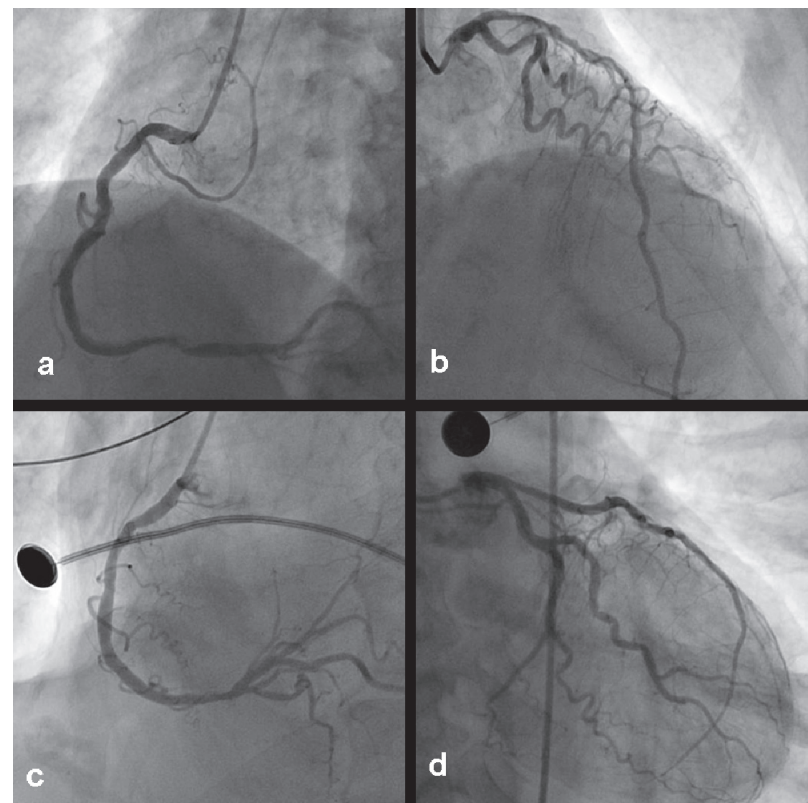

Figure 2 In case one, noncritical, distal lesions of the right coronary artery (RCA) (a) and noncritical atherosclerotic lesions of the left main coronary artery (b) did not correspond with the wall motion abnormality observed on ventriculography. In case two, there was no evidence of coronary arterial stenosis of either the RCA (c) or the left anterior descending artery (d).

of transient left ventricular apical ballooning syndrome (takotsubo cardiomyopathy), the consensus was that both patients had clinical presentations most consistent with takotsubo cardiomyopathy (Bybee et al 2004).

The cases are somewhat atypical in that both occurred in men and neither patient complained of chest pain. However, both patients had prominent, diffuse ST segment elevations on ECG, the most common ECG finding in takotsubo cardiomyopathy. New Q-waves have been observed in up to $31 \%$ of patients, and in some instances, the Q-waves have been a transient phenomenon (Bybee et al 2004). As illustrated in Figure 3, the second patient did have Q-waves on presentation. In this case, the Q-waves persisted despite recovery of ventricular function. Figures $4 \mathrm{a}$ and $4 \mathrm{~b}$

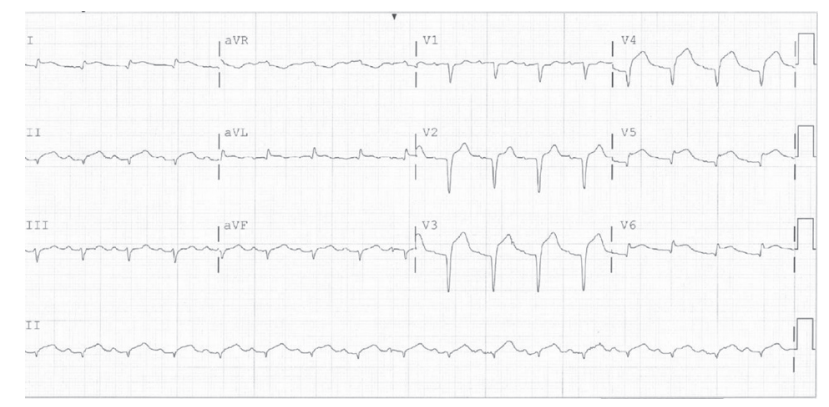

Figure 3 In the second case, the ECG was notable for new Q-waves in inferior and precordial leads as well as ST elevation suggestive of acute ischemia or injury in the anterior-septal leads. 


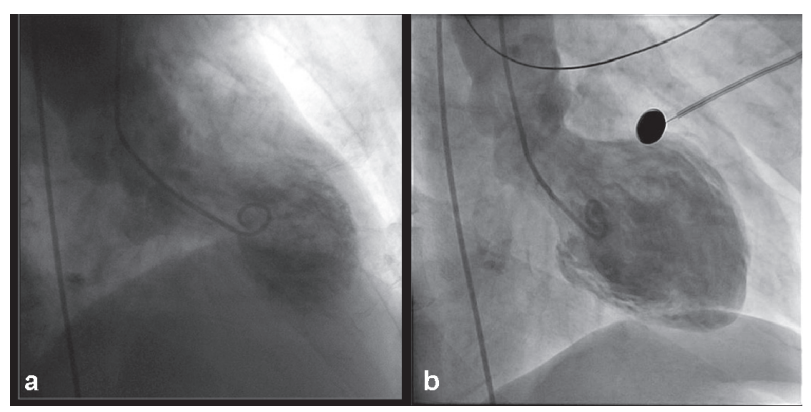

Figure 4 (a) This image is a left ventriculogram obtained during systole in the first patient. Hypercontractility of the basal segment and akinesis of the apical segment create the apical ballooning effect. (b) The ventriculogram obtained in the second case also demonstrates apical ballooning.

show clearly that both patients had significant left ventricular dysfunction. In Figure $4 \mathrm{a}$, there appears to be some degree of asymmetry in the balloon effect. While takotsubo cardiomyopathy is classically described as apical ballooning, cases of midventricular ballooning have been reported. Although the exact mechanism is unclear, it is believed that the pathophysiology underlying transient midventricular ballooning is the same as that of takotsubo cardiomyopathy (Hurst et al 2006). In the second case, Figure 4b, the ventricular ballooning is more classical in distribution, more closely resembling the octopus trap. In both cases, the affected area is not related to a single coronary artery distribution, which supports an etiology other than coronary artery disease. This is a hallmark feature of takotsubo cardiomyopathy. Another prominent feature is the recovery of ventricular function within weeks. Both patients demonstrated recovery of myocardial function within 30 days.

These cases are notable for their occurrence in men and the association with treatment of metastatic cancer including bevacizumab. The cases described, animal models and data from clinical trials implicate bevacizumab as a potential cause of takotsubo cardiomyopathy in some patients. Although the exact mechanism of the suspected cardiotoxicity remains unknown, the discovery of this side effect may provide insights on the pathogenesis of takotsubo cardiomyopathy.

\section{Conclusion}

Bevacizumab is a novel chemotherapeutic agent, which inhibits VEGF - a promising target for anti-neoplastic agents. We have presented two cases of takotsubo cardiomyopathy, which developed after exposure to bevacizumab. The exact nature of the relationship between exposure to bevacizumab, the subsequent VEGF inhibition, and takotsubo cardiomyopathy remains to be seen. Further studies regarding the potential tonic arterioprotective effects of VEGF and the potential role of VEGF inhibition in the pathophysiology of takotsubo cardiomyopathy are warranted.

\section{Disclosures}

The authors have no conflicts of interest to disclose.

\section{References}

Dote K, Sato H, Tateishi H, Uchida T, Ishihara M. 1991. Myocardial stunning due to simultaneous multivessel coronary spasms: a review of 5 cases. J Cardiol, 21:203-14.

Drimal J, Zurova-Nedelcevova J, Knezl V, Sotnikova R, Navarova J. 2006. Cardiovascular toxicity of the first line cancer chemotherapeutic agents: doxorubicin, cyclophosphamide, streptozocin and bevacizumab. Neuro Endocrinol Lett, 27(Suppl 2):176-9.

Genentech, Inc. 2008. Bevacizumab Prescribing Information [online]. Accessed 23 May 2008. URL: http://www.gene.com/gene/products/ information/pdf/avastin-prescribing.pdf.

Hurst R, Askew J, Reuss C, et al. 2006. Transient midventricular ballooning syndrome. J Am Coll Cardiol, 48:579-83.

Hurwitz H, Fehrenbacher L, Novotny W, et al. 2004. Bevacizumab plus irinotecan, fluorouracil and leucovorin for metastatic colorectal Cancer. N Engl J Med, 350:2335-42.

Izumiya Y, Shiojima I, Sato K, Sawyer D, Colussi W, Walsh K. 2006. Vascular endothelial growth factor blockade promotes the transition from compensatory cardiac hypertrophy to failure in response to pressure overload. Hypertension, 47:887-93.

Sandler A, Gray R, Perry MC, et al. 2006. Paclitaxel-carboplatin alone or with bevacizumab for non-small-cell lung cancer. $N$ Engl J Med, 355: $2542-50$

Scappaticci F, Skillings J, Holden S, et al. 2007. Arterial thromboembolic events in patients with metastatic carcinoma treated with chemotherapy and bevacizumab. $J$ Nat Cancer Inst, 99:1232-9.

Traina T, Rugo H, Dickler M. 2007. Bevacizumab for Advanced Breast Cancer. Hematol Oncol Clin N Am, 21:303-19.

Van De Walle S, Gevaert S, Gheerhaert P, et al. 2006. Transient stress-induced cardiomyopathy with an "inverted takotsubo" contractile pattern. Mayo Clin Proc, 81:1499-502.

Zachary I, Martin J. 2006. Viewpoint: an explanation for the cardiovascular effects of bevacizumab and rofecoxib? Circulation: European Perspectives, 7: f173-f5. 